\title{
Response of RNA polymerase to ppGpp: requirement for the $\omega$ subunit and relief of this requirement by DksA
}

\author{
Catherine E. Vrentas, ${ }^{1}$ Tamas Gaal, ${ }^{1}$ Wilma Ross, ${ }^{1}$ Richard H. Ebright, ${ }^{2}$ and Richard L. Gourse ${ }^{1,3}$ \\ ${ }^{1}$ Department of Bacteriology, University of Wisconsin-Madison, Madison, Wisconsin 53706, USA; ${ }^{2}$ Howard Hughes Medical \\ Institute, Waksman Institute, and Department of Chemistry, Rutgers University, Piscataway, New Jersey 08854, USA
}

Previous studies have come to conflicting conclusions about the requirement for the $\omega$ subunit of RNA polymerase in bacterial transcription regulation. We demonstrate here that purified RNAP lacking $\omega$ does not respond in vitro to the effector of the stringent response, ppGpp. DksA, a transcription factor that works in concert with ppGpp to regulate rRNA expression in vivo and in vitro, fully rescues the ppGpp-unresponsiveness of RNAP lacking $\omega$, likely explaining why strains lacking $\omega$ display a stringent response in vivo. These results demonstrate that $\omega$ plays a role in RNAP function (in addition to its previously reported role in RNAP assembly) and highlight the importance of inclusion of $\omega$ in RNAP purification protocols. Furthermore, these results suggest that either one or both of two short segments in the $\beta^{\prime}$ subunit that physically link $\omega$ to the ppGpp-binding region of the enzyme may play crucial roles in ppGpp and DksA function.

[Keywords: Transcription initiation; RNAP structure; rRNA promoters; stringent response; $\omega$ ]

Received June 6, 2005; revised version accepted July 22, 2005.

In Escherichia coli, transcription is carried out by a multi-subunit RNA polymerase (RNAP) composed of six subunits, including two copies of $\alpha$ and one copy each of $\beta, \beta^{\prime}, \sigma$, and $\omega$ (for a recent review, see Geszvain and Landick 2004). $\alpha_{2}, \beta, \beta^{\prime}$, and $\omega$ comprise core RNAP, which is catalytically active but unable to recognize promoters. The $\alpha_{2}$ dimer serves as the scaffold on which $\beta$ and $\beta^{\prime}$ assemble. $\beta$ and $\beta^{\prime}$ make up the vast majority of RNAP by mass and create the enzyme's active center. To initiate transcription, one of several types of $\sigma$ subunits, most commonly $\sigma^{70}$, binds to core to form RNAP holoenzyme. $\sigma$ and $\alpha$ are site-specific DNA-binding proteins that account for specific promoter recognition. Although a high-resolution structure of $E$. coli RNAP has not yet been determined, X-ray structures of the Thermus aquaticus and Thermus thermophilus holoenzymes (Murakami et al. 2002b; Vassylyev et al. 2002), as well as of a T. aquaticus RNAP holoenzyme-DNA complex (Murakami et al. 2002a), elucidate how the RNAP subunits interact with each other and with template DNA.

$\omega$, encoded by the E. coli rpoZ gene, is the smallest RNAP subunit at only $10 \mathrm{kDa}$. $\omega$ has homologs in all three kingdoms of life. It is present in all sequenced freeliving bacteria (although some intracellular parasitic

${ }^{3}$ Corresponding author.

E-MAIL rgourse@bact.wisc.edu; FAX (608) 262-9865.

Article and publication are at http://www.genesdev.org/cgi/doi/10.1101/ gad. 1340305 . bacteria, such as Chlamydia sp., appear to lack an $\omega$ homolog), in archaea (RpoK), and in eukaryotes (RPB6) (Minakhin et al. 2001). The RNAP structures indicate that there is one copy of $\omega$ per RNAP, and that it interacts with $\beta^{\prime}$ conserved regions $D$ and $G$ and wraps over and around the $\beta^{\prime}$ C-terminal tail, latching $\beta^{\prime}$ to the $\alpha_{2} \beta$ subassembly (Minakhin et al. 2001). The RNAP structures therefore are consistent with the model that $\omega$ functions as a chaperone in enzyme assembly by facilitating the binding of $\beta^{\prime}$ to $\alpha_{2} \beta$ (Gentry and Burgess 1993; Mukherjee et al. 1999; Ghosh et al. 2001, 2003). In support of this view, reconstitution of RNAP from its individual subunits is less efficient in the absence of $\omega$ (Mukherjee and Chatterji 1997).

In contrast to the insights that the structures of RNAP provide about a role for $\omega$ in enzyme assembly, the structures do not suggest an obvious role for $\omega$ in enzyme function. Furthermore, no defects have been reported in the specific activity of RNAP lacking $\omega$ or in the interactions of $\omega$-deficient RNAP with transcription factors. Indeed, functional RNAP is produced by standard in vitro reconstitution procedures that do not include $\omega$ (Tang et al. 1995), and overproduction of core RNAP in vivo without co-overproduction of $\omega$ has been used extensively as a method for producing homogeneous mutant RNAP for structure-function analysis of RNAP in vitro (Artsimovitch et al. 2003, 2004).

In spite of the extraordinary evolutionary conservation of $\omega$ and its role in RNAP assembly, E. coli mutants 
lacking $r p o Z$ are viable. Some $r p o Z$ mutants grow more slowly than wild type (Mukherjee and Chatterji 1997), but this phenotype has been proposed to result from polar effects on the downstream gene spoT (Gentry and Burgess 1989). SpoT is a bifunctional enzyme that can both synthesize and degrade the global transcriptional regulator guanosine 5 '-diphosphate 3 '-diphosphate, ppGpp (Xiao et al. 1991). ppGpp (used here to refer to both the tetraphosphate and its pentaphosphate precursor) is synthesized by the RelA and SpoT proteins in response to nutrient starvation (Cashel et al. 1996).

ppGpp strongly and directly inhibits promoters for ribosomal RNA (rRNA) and transfer RNA (tRNA) in vivo (Barker et al. 2001b; Paul et al. 2004a,b; Gralla 2005). In addition, ppGpp both directly and indirectly stimulates a set of promoters that make transcripts coding for enzymes for amino acid biosynthesis and transport (Barker et al. 2001a; Paul et al. 2005). The 151-amino-acid DksA protein binds directly to RNAP and is required for both negative control of rRNA promoters and positive control of amino acid promoters in response to changing concentrations of ppGpp in vivo (Paul et al. 2004a, 2005; Perederina et al. 2004). Thus, ppGpp and DksA together (ppGpp/DksA) result in a global response to amino acid starvation referred to as the stringent response.

Ishihama and colleagues (Igarashi et al. 1989) found that separation of $\omega$ from core enzyme by partial urea denaturation appeared to result in RNAP that was insensitive to ppGpp when assayed by transcription in vitro. Addition of refolded $\omega$ resulted in partial inhibition by ppGpp. These results, in conjunction with the colocation of rpoZ in the same operon as spoT, led to the conclusion that there was a functional link between $\omega$ and ppGpp. This conclusion, however, was contradicted by the observation that cells lacking rpoZ still displayed stringent regulation of rRNA transcription in response to amino acid starvation (Gentry et al. 1991). Furthermore, the link between ppGpp and $\omega$ was further clouded by the limited availability at that time of assays for examining effects of ppGpp in vitro, by subsequent reports that other polypeptides were present in some $\omega$ preparations (Mukherjee and Chatterii 1997), and by the observation that RNAP lacking $\omega$ was generally less active than wildtype RNAP (Mukherjee and Chatterji 1997). Finally, the positions of ppGpp and DksA in the RNAP holoenzyme indicate that neither ppGpp nor DksA is located in the immediate vicinity of $\omega$ (Artsimovitch et al. 2004; Perederina et al. 2004; I. Toulokhonov, J. Mukhopadhyay, R.H. Ebright, and R.L. Gourse, unpubl.; see Discussion).

The details of the mechanism by which ppGpp and DksA exert their effects on transcription initiation are still ill-defined. However, we have proposed that ppGpp/ DksA functions, at least in part, by lowering the energy of a transition state on the pathway to formation of the open complex, the promoter complex in which the DNA around the transcription start site is unwound to accommodate polymerization of NTPs into RNA. Transcription initiation is a multistep process in which RNAP (R) first binds to the promoter $(\mathrm{P})$ to form a closed complex $\left(R P_{C}\right)$ and then proceeds through a series of kinetic in- termediates to form the open complex $\left(\mathrm{RP}_{\mathrm{O}}\right) \cdot \mathrm{ppGpp} /$ DksA decreases the lifetimes of competitor-resistant complexes at all promoters that have been examined. The short lifetime of the competitor-resistant rRNA promoter complex is rate-limiting for transcription. Therefore, we have proposed that inhibition of rRNA transcription by ppGpp/DksA results from shifting the equilibrium from this complex to an earlier intermediate before condensation of the initial phosphodiester bond(s) can occur (Barker et al. 2001b; Paul et al. 2004a, 2005).

Promoters positively regulated by ppGpp/DksA make intrinsically long-lived competitor-resistant complexes, such that the reduced lifetime of these complexes by ppGpp/DksA is not rate-determining for transcription (Barker et al. 2001a; Paul et al. 2005). Rather, these promoters are rate-limited by $\mathrm{RP}_{\mathrm{O}}$ formation. We have proposed that by lowering the energy of a transition state, ppGpp/DksA thereby increases the rate of $\mathrm{RP}_{\mathrm{O}}$ formation directly. In this model, ppGpp/DksA would stimulate transcription from promoters that are slow to form open complexes, but whose open complexes are longlived once formed.

In the process of analysis of amino acid residues in RNAP required for ppGpp function, we purified core RNAP by co-overproduction of the $\alpha, \beta$, and $\beta^{\prime}$ subunits in vivo, reconstituted these enzymes with $\sigma$, and observed that even wild-type holoenzyme failed to respond to ppGpp in vitro. We demonstrate conclusively here that addition of $\omega$, either before or after assembly of the core enzyme, restores RNAP to its native state with respect to regulation by ppGpp. We further demonstrate that DksA eliminates the $\omega$ requirement for the response of RNAP to ppGpp, resolving the discrepancy between previous conclusions about the requirement for $\omega$ for ppGpp function in vitro versus in vivo. In addition to their importance for methodological purposes, these results provide important insights about the mechanism of action of ppGpp/DksA.

\section{Results}

RNAP produced by overproduction of core subunits in vivo is insensitive to $p p G p p$

ppGpp decreases multiple-round transcription from $\operatorname{rrn} B$ P1 by native E. coli E $\sigma^{70}$ RNAP holoenzyme by two- to threefold (Fig. 1A, "native RNAP"; Table 1, rows 1 and 2; Barker et al. 2001b). ppGpp also decreases the half-life (increases the dissociation constant, $k_{d}$ ) of competitorresistant complexes formed on $\operatorname{rrn} B$ P1 by two- to threefold (Fig. 1B; Table 1, rows 1 and 2; Barker et al. 2001b). However, when holoenzyme was purified by co-overproduction of $\alpha, \beta$, and $\beta^{\prime}$ in vivo (Artsimovitch et al. 2004), and this core enzyme was reconstituted with $\sigma$ in vitro (hereafter referred to as "overproduced $\alpha_{2} \beta \beta^{\prime}$ RNAP"), this RNAP did not respond normally to ppGpp (Fig. 1C,D; Table 1, row 5). Specifically, transcription by overproduced $\alpha_{2} \beta \beta^{\prime}$ RNAP was inhibited slightly if at all by ppGpp, and the lifetime of overproduced $\alpha_{2} \beta \beta^{\prime}$ RNAP complexes was the same in the presence and absence of 

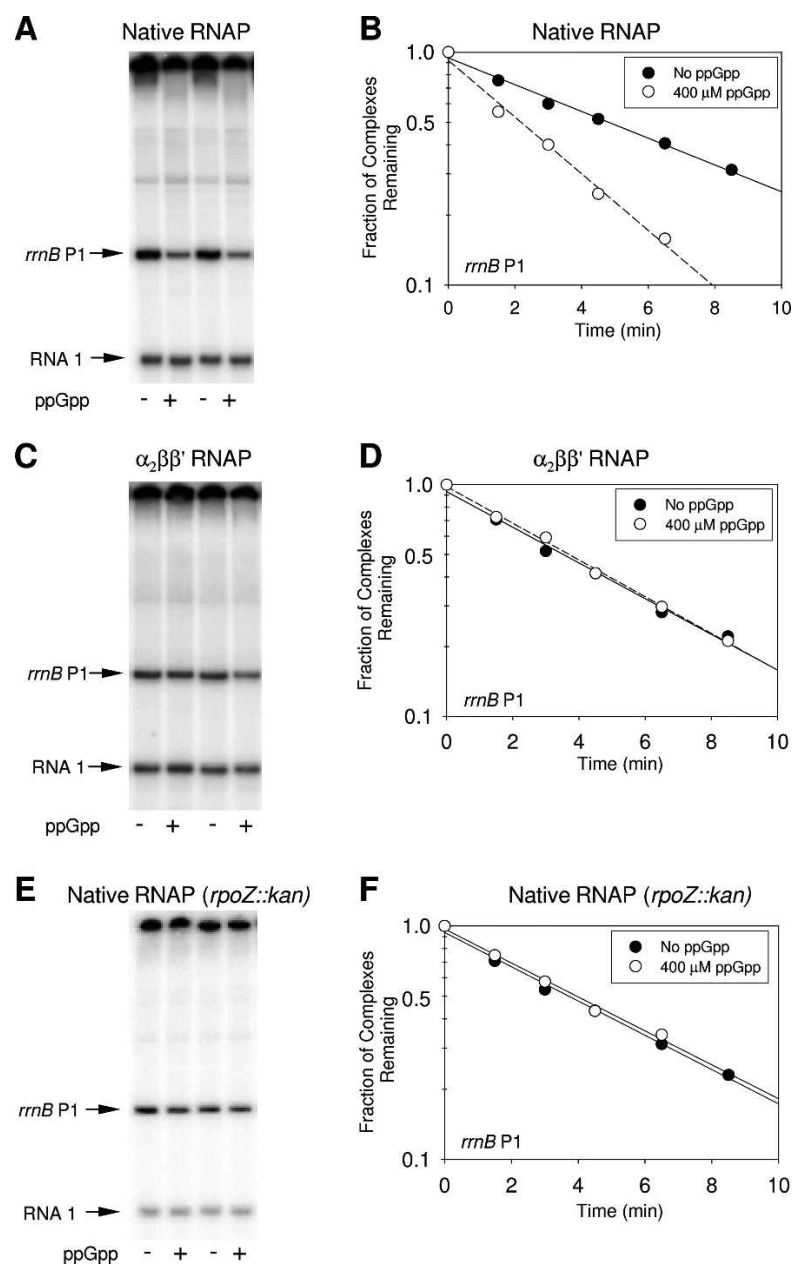

Figure 1. RNAP made from overproduced or native core lacking $\omega$ is unresponsive to ppGpp. $(A, B)$ Native RNAP purified as holoenzyme without overproduction of core subunits. $(C, D)$ Core RNAP made from strain overproducing $\alpha_{2} \beta \beta^{\prime}$, and then reconstituted with $\sigma$ to make holoenzyme. $(E, F)$ Native RNAP purified as holoenzyme from a strain lacking $\omega$. $(A, C, E)$ Multiple-round transcription in buffer (see Materials and Methods) containing $170 \mathrm{mM} \mathrm{NaCl} \pm 400 \mu \mathrm{M}$ ppGpp. The supercoiled plasmid template contained both the $\operatorname{rrnB}$ P1 promoter and the RNA 1 promoter (see Materials and Methods). Each gel contains duplicate lanes. Quantitation of inhibition by ppGpp (mean and standard deviation from multiple independent experiments, -ppGpp/+ppGpp) is provided in Table $1 .(B, D, F)$ RNAP-promoter lifetimes $\pm 400 \mu \mathrm{M} p \mathrm{pgpp}$, determined by a transcription-based assay on the same templates as in $A, C$, and $E$, using transcription buffer containing $30 \mathrm{mM} \mathrm{KCl}$ (see Materials and Methods). The fraction of competitor-resistant complexes remaining is pictured on a semilog plot as a function of time after heparin addition (30 sec before time zero). Reactions without ppGpp (filled circles) and with ppGpp (open circles) were measured in parallel. The fraction remaining was normalized to 1.0 at $0 \mathrm{sec}$. Averages and standard deviations of the ratios of the resulting dissociation constants $\left(k_{d}\right)$ for each RNAP from multiple independent experiments are provided in Table 1.

ppGpp. We found that other RNAP preparations made by overproduction of $\alpha_{2} \beta \beta^{\prime}$ and purified using a chitin affinity tag also were insensitive to ppGpp (data not shown).
Native RNAP lacking the w subunit is insensitive to $p p G p p$

Two explanations potentially could account for the inability of overproduced $\alpha_{2} \beta \beta^{\prime}$ RNAP to respond to ppGpp: Overproduction might result in a ppGpp-unresponsive conformation of the enzyme, or overproduced RNAP might be missing some necessary factor.

Since a previous report suggested that $\omega$ might play a role in the response to ppGpp (Igarashi et al. 1989), and since overproduced RNAP lacked $\omega$, we tested the effect of ppGpp on native RNAP purified from a strain lacking rpoZ. RNAP purified from an rpoZ::kan strain (Gentry et al. 1991) was unresponsive to ppGpp in both the transcription and RNAP-promoter lifetime assays (Fig. 1E,F; Table 1, row 3 ), strongly suggesting that the unresponsiveness of overproduced $\alpha_{2} \beta \beta^{\prime}$ RNAP to ppGpp resulted from the absence of $\omega$. E. coli RNAP reconstituted in vitro from individually purified $\alpha, \beta, \beta^{\prime}$, and $\sigma$ subunits was also insensitive to ppGpp (data not shown). Furthermore, the native RNAP preparations from the wildtype and rpoZ::kan strains were purified in the form of holoenzyme, in contrast to the overproduced RNAP preparations for which $\sigma$ was added to core in vitro. Thus, reconstitution of core RNAP with $\sigma$ in vitro was not responsible for the inability of RNAP lacking $\omega$ to respond to ppGpp (cf. Berghofer-Hochheimer et al. 2005).

Co-overproduction of $\omega$ with the other core subunits in vivo or reconstitution of $\omega$ with overproduced $\alpha_{2} \beta \beta^{\prime}$ $R N A P$ in vitro restores sensitivity to $p p G p p$

We tested whether co-overproduction of $\omega$ with overproduced $\alpha_{2} \beta \beta^{\prime}$ core RNAP in vivo (to make overproduced $\alpha_{2} \beta \beta^{\prime} \omega$ RNAP) would restore sensitivity to ppGpp by overproduced RNAP. Transcription from $\operatorname{rrnB}$ P1 by overproduced $\alpha_{2} \beta \beta^{\prime} \omega$ RNAP was inhibited 3.6-fold by ppGpp (Fig. 2A; Table 1, row 4), and competitor-resistant complexes were 2.9-fold shorter-lived in the presence of ppGpp (Fig. 2B; Table 1, row 4). Thus, in contrast to overproduced $\alpha_{2} \beta \beta^{\prime}$ RNAP, overproduced $\alpha_{2} \beta \beta^{\prime} \omega$ RNAP responded to ppGpp. In the absence of ppGpp, the dissociation constants of RNAP complexes containing overproduced $\alpha_{2} \beta \beta^{\prime}$ (Fig. 1D; Table 1, row 5) or overproduced $\alpha_{2} \beta \beta^{\prime} \omega$ RNAP (Fig. 2B; Table 1, row 4), measured in parallel on $r r n B$ P1, were virtually identical, indicating that $\omega$ did not affect the intrinsic lifetime of the RNAP-promoter complex.

In the experiments reported above (Fig. 2A,B), $\omega$ coassembled with core RNAP in vivo before purification. In Figure $2 \mathrm{C}$ and $\mathrm{D}$, we tested whether purified $\omega$ added in vitro to preassembled core RNAP, followed by addition of $\sigma$ (overproduced $\alpha_{2} \beta \beta^{\prime}+\omega$ RNAP), would restore ppGpp sensitivity to RNAP. ppGpp inhibited transcription by the resulting holoenzyme 3.0-fold (Fig. 2C; Table 1 , row 6), and it reduced the lifetime of the promoterRNAP complex 2.7-fold (Fig. 2D; Table 1, row 6). Thus, inclusion of $\omega$, either by co-overproduction with other core subunits in vivo (Fig. 2A,B), or by addition of $\omega$ to 
Table 1. Effects of $\omega$ and $p p G p p$ on transcription inhibition and promoter complex dissociation

\begin{tabular}{lccccc}
\hline RNAP & $\begin{array}{c}\text { Presence } \\
\text { of } \omega\end{array}$ & $\begin{array}{c}-\mathrm{ppGpp} / \\
+\mathrm{ppGpp}^{\mathrm{g}}\end{array}$ & $\begin{array}{c}k_{d} \times 10^{4}\left(\mathrm{sec}^{-1}\right) \\
(-\mathrm{ppGpp})^{\mathrm{h}}\end{array}$ & $\begin{array}{c}k_{d} \times 10^{4}\left(\mathrm{sec}^{-1}\right) \\
(+\mathrm{ppGpp})^{\mathrm{h}}\end{array}$ & $\begin{array}{c}\left(k_{d}+\mathrm{ppGpp}\right) / \\
\left(k_{d}-\mathrm{ppGpp}\right)\end{array}$ \\
\hline Native holoenzyme $^{\mathrm{a}}$ & + & $2.5 \pm 0.5$ & 0.24 & 0.53 & $2.2 \pm 0.36$ \\
Native holoenzyme & + & $2.8 \pm 0.3$ & $0.15^{\mathrm{i}}$ & $0.43^{\mathrm{i}}$ & $2.9^{\mathrm{i}}$ \\
Native $(r p o Z:: \text { kan })^{\mathrm{c}}$ & - & $1.1 \pm 0.03$ & 0.31 & 0.28 & $0.9 \pm 0.05$ \\
Overproduced $\alpha_{2} \beta \beta^{\prime} \omega^{\mathrm{d}}$ & + & $3.6 \pm 0.5$ & 0.32 & 0.94 & $2.9 \pm 0.09$ \\
Overproduced $\alpha_{2} \beta \beta^{\prime \mathrm{e}}$ & - & $1.3 \pm 0.2$ & 0.31 & 0.31 & $1.0 \pm 0.05$ \\
Overproduced $\alpha_{2} \beta \beta^{\prime}+\omega^{\mathrm{f}}$ & + & $3.0 \pm 0.4$ & 0.15 & 0.41 & $2.7 \pm 0.19$ \\
\hline
\end{tabular}

${ }^{a}$ Native RNAP holoenzyme purified by method of Burgess and Jendrisak (1975).

${ }^{\text {b}}$ Native RNAP holoenzyme purified by immunoaffinity chromatography.

${ }^{\mathrm{c}}$ Native RNAP holoenzyme purified from rpoZ::kan strain by immunoaffinity chromatography.

${ }^{\mathrm{d}}$ Core RNAP purified by overproduction of $\alpha_{2} \beta \beta^{\prime}$ and $\omega$, reconstituted in vitro with purified $\sigma$.

'Core RNAP purified by overproduction of $\alpha_{2} \beta \beta^{\prime}$, reconstituted in vitro with purified $\sigma$.

${ }^{\mathrm{f}}$ Core RNAP purified by overproduction of $\alpha_{2} \beta \beta^{\prime}$, reconstituted in vitro with purified $\sigma$ and $\omega$.

${ }^{\mathrm{g}}$ Transcription was performed as described in Figure 1 and Materials and Methods. The ratio is the amount of transcription from the $r r n B$ P1 promoter \pm ppGpp. The ratio provided in row 2 is the mean and range from two experiments. All other means and standard deviations are from at least three experiments.

${ }^{\mathrm{h}} k_{d}$ values were calculated as described in Materials and Methods; representative assays are presented in Figures 1-4. Absolute $k_{d}$ values varied slightly from day to day, most likely from slight changes in solution conditions. Effects of ppGpp were always compared directly in the same experiment. Enzymes produced with and without $\omega$ by overproduction (rows 4 and 5) were also compared directly in the same experiment. Associated errors represent standard deviations or ranges as appropriate from two or more experiments.

${ }^{i}$ The half-life \pm ppGpp of the native holoenzyme purified by immunoaffinity chromatography was determined only once.

preassembled purified core RNAP lacking $\omega$, restores the ability of RNAP to respond to ppGpp.

\section{Insensitivity of overproduced RNAP lacking $\omega$ to $p p G p p$ also occurs on non-rRNA promoters}

ppGpp reduces the lifetimes of RNAP complexes on all promoters, although it inhibits transcription only from promoters that make intrinsically short-lived complexes (Barker et al. 2001b). We tested whether $\omega$ affected RNAP's insensitivity to ppGpp on a different promoter by comparing the behavior of overproduced $\alpha_{2} \beta \beta^{\prime}$ RNAP and overproduced $\alpha_{2} \beta \beta^{\prime} \omega$ RNAP on the lacUV5 promoter. ppGpp reduced the lifetime of the lacUV5 promoter complex containing $\omega$ 3.8-fold (Fig. 3A), but had little or no effect on the complex formed without $\omega$ (1.2fold decrease) (Fig. 3B). Thus, the $\omega$ requirement for a response to $\mathrm{ppGpp}$ is not promoter-specific.

\section{$\omega$ is not required for DksA function}

DksA directly reduces the lifetimes of promoter complexes formed with RNAP, directly inhibits transcription from rRNA promoters, and greatly increases the effects of ppGpp in vivo and in vitro (Paul et al. 2004a). Therefore, we next determined whether DksA function, like ppGpp function, requires $\omega$ (Fig. 4). Transcription from $r r n B$ P1 using enzymes purified by overproduction of core with and without co-overproduction of $\omega\left(\alpha_{2} \beta \beta^{\prime} \omega\right.$ and $\alpha_{2} \beta \beta^{\prime}$ RNAP, respectively) was inhibited similarly by DksA at each of several DksA concentrations tested (Fig. 4A, cf. black and gray bars). Likewise, $\omega$ did not affect the ability of DksA to reduce the lifetimes of $\operatorname{rrn} B$ P1-RNAP complexes (Fig. 4B) or lacUV5-RNAP complexes (Fig. 4C).

\section{RNAP lacking $\omega$ but containing DksA responds to $p p G p p$}

Previous studies showed that DksA and ppGpp work synergistically to inhibit transcription from rRNA promoters (Paul et al. 2004a). Since RNAP nevertheless responds to ppGpp in strains lacking rpoZ (Gentry et al. 1991), we tested whether DksA might be able to restore ppGpp responsiveness to an RNAP preparation lacking $\omega . \operatorname{rrn} B$ Pl activity was measured using solution conditions in which ppGpp or DksA by themselves have little or no effects on transcription, but the two together severely inhibit transcription from $\operatorname{rrn} B \mathrm{P} 1$ by native RNAP (Paul et al. 2004a). Figure 5A shows that when both DksA and ppGpp were present, transcription by both overproduced $\alpha_{2} \beta \beta^{\prime}$ and $\alpha_{2} \beta \beta^{\prime} \omega$ RNAP was strongly inhibited. The DksA concentration dependence of this inhibition (at $400 \mu \mathrm{M}$ ppGpp) was similar for both RNAP preparations. DksA also rescued the ppGpp insensitivity of native $\alpha_{2} \beta \beta^{\prime}$ RNAP made from the rpoZ::kan strain (data not shown).

To obtain a quantitative estimate of the ability of DksA to rescue the responsiveness of $\alpha_{2} \beta \beta^{\prime}$ RNAP to ppGpp, we measured the half-lives of RNAP-lacUV5 promoter complexes at a range of ppGpp concentrations (and $500 \mathrm{nM}$ DksA). The ppGpp concentration dependence of the effect of DksA and ppGpp together on halflife was indistinguishable for the overproduced $\alpha_{2} \beta \beta^{\prime}$ and $\alpha_{2} \beta \beta^{\prime} \omega$ RNAP preparations (Fig. $5 \mathrm{~B}$ ).

We conclude that DksA restores ppGpp responsiveness to RNAP lacking $\omega$, and that this most likely accounts for the ability of the rpoZ mutant strain to maintain a stringent response (Gentry et al. 1991). Strains lacking both $r p o Z$ and $d k s A$ therefore should not exhibit a stringent response. We constructed the $\Delta r p o Z \Delta d k s A$ mutant, but we could not easily assess further loss of 
A

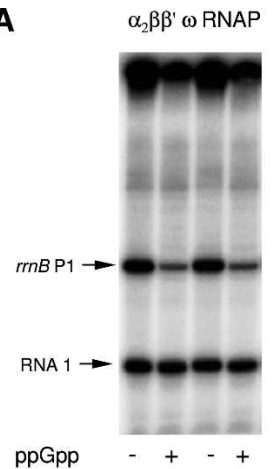

C $\alpha_{2} \beta \beta^{\prime}$ RNAP + $\omega$
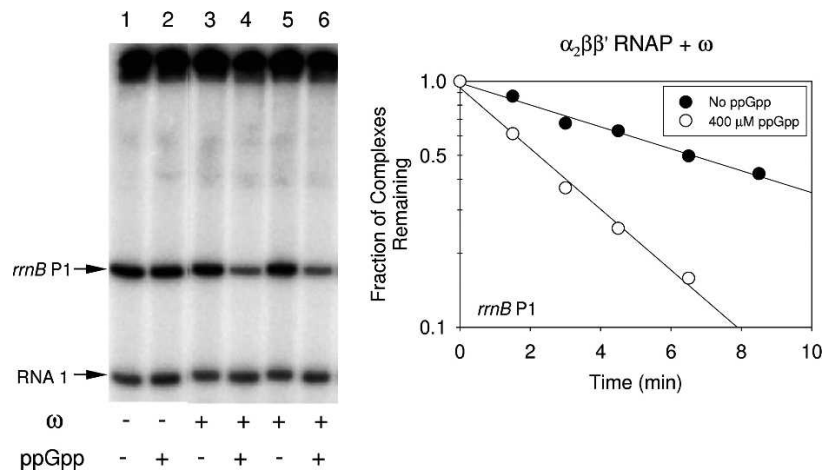

Figure 2. $\omega$ restores ppGpp sensitivity to overproduced core RNAP. Templates and methods as described in Figure 1. $(A, B)$ RNAP made by overproduction of $\alpha_{2} \beta \beta^{\prime}$ and $\omega$ followed by addition of purified $\sigma$ in vitro. $(C, D)$ RNAP made by overproduction of $\alpha_{2} \beta \beta^{\prime}$ followed by addition of purified $\omega$ and $\sigma$ in vitro. $(A, C)$ Multiple-round transcription; $400 \mu \mathrm{M}$ ppGpp and/or $\omega$ included as indicated. Quantitation (means and standard deviations from multiple independent experiments, -ppGpp/ + ppGpp) is provided in Table 1 . $(B, D)$ RNAP-promoter lifetimes. Means and standard deviations of the resulting dissociation constants $\left(k_{d}\right)$ for each RNAP from multiple independent experiments are provided in Table 1 . The experiments reported in $B$ and $D$ were not performed in the exact same buffer, and slight differences in solution conditions, rather than the methods by which the holoenzymes were made, account for the differences in the intrinsic dissociation constants $\left(0.32 \times 10^{-4} \mathrm{vs}\right.$. $0.15 \times 10^{-4} \mathrm{sec}^{-1}$; see Materials and Methods).

rRNA regulation in this double mutant, since rRNA transcription is already derepressed in strains lacking $d k s A$ alone (Paul et al. 2004a).

ppGpp not only inhibts rRNA promoters, but it also activates amino acid promoters (Paul et al. 2005). Strains lacking $d k s A$ alone are defective in transcription from a subset of amino acid promoters and display complex amino acid requirements, because these promoters are unable to respond normally to ppGpp (Paul et al. 2005). As expected, strains lacking both rpoZ and $d k s A$ also displayed complex amino acid requirements (data not shown), strongly suggesting that they are unable to respond normally to ppGpp. Interestingly, the double mutant also displayed additional nutritional requirements compared with the strain lacking $d k s A$ alone. Further studies will be needed to address the basis for these phenotypes.

\section{Discussion}

$\omega$ plays a role in RNAP function

We demonstrate here that RNAP holoenzymes lacking $\omega$ have a defect in function: They are completely unable to respond to ppGpp. Thus, the absence of $\omega$ might also compromise other properties of RNAP. In fact, preliminary data suggest that RNAP lacking $\omega$ associates more slowly than wild-type RNAP with the $\lambda P_{R}$ promoter (R. Saecker and M.T. Record Jr., pers. comm.; see also Mukherjee and Chatterii 1997). Caution therefore should be exercised in interpreting results obtained with RNAP lacking $\omega$.

We also show here that DksA, a protein recently discovered to work synergistically with ppGpp in vitro and in vivo (Paul et al. 2004a, 2005), suppresses the deficiency of RNAP lacking $\omega$ to respond to ppGpp in vitro. Since strains lacking $r p o Z$ nevertheless still respond to ppGpp (Gentry et al. 1991), the presence of DksA in rpoZ mutant cells in vivo likely explains the previous discrepancy between the apparent requirement for $\omega$ for RNAP
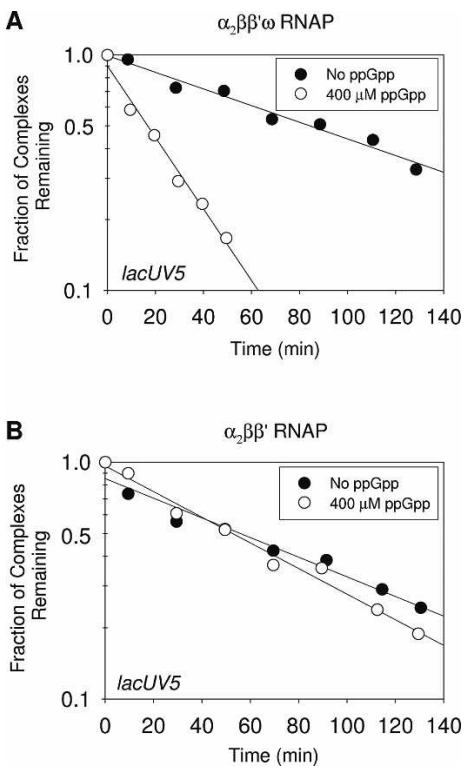

Figure 3. Insensitivity to ppGpp of RNAP lacking $\omega$ is not promoter-specific. The template was a DNA fragment containing the lacUV5 promoter. Half-lives were determined by filterbinding in transcription buffer containing $100 \mathrm{mM} \mathrm{KCl}$ in the absence (filled circles) and presence (open circles) of $400 \mu \mathrm{M}$ ppGpp. Representative experiments are shown. (A) RNAP made by overproduction of core $\left(\alpha_{2} \beta \beta^{\prime}\right.$ and $\left.\omega\right)$ followed by reconstitution with $\sigma$. The ratio of the complex lifetime with ppGpp/ without ppGpp was $3.8 \pm 0.7$ (four independent assays). (B) RNAP made by overproduction of core $\left(\alpha_{2} \beta \beta^{\prime}\right)$ without $\omega$ followed by reconstitution with $\sigma$. The ratio of the complex halflife with ppGpp/without ppGpp was $1.2 \pm 0.1$ (four independent assays). 
A

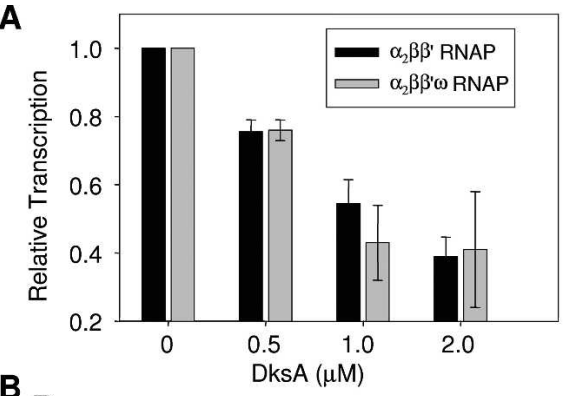

B
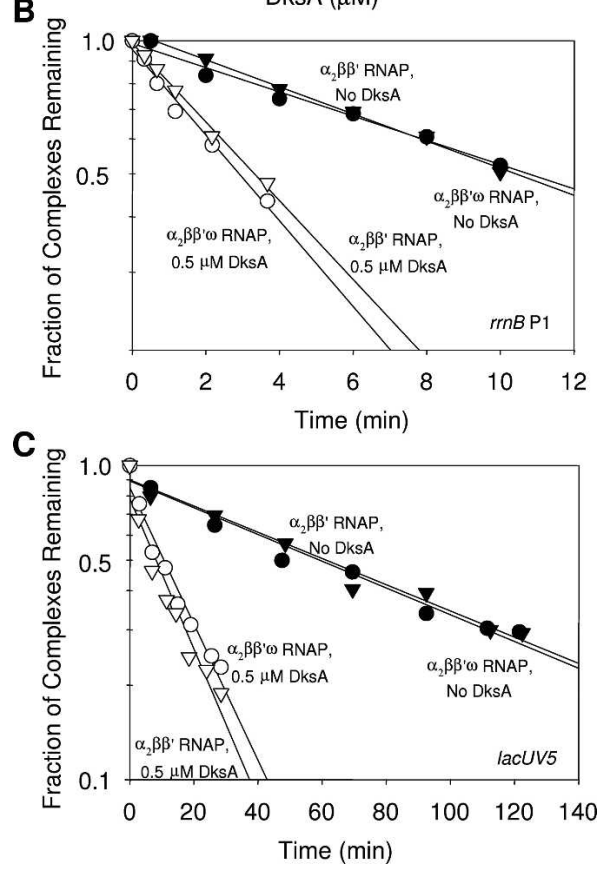

Figure 4. $\omega$ is not required for DksA function. $(A)$ Single-round transcription from $r r n B$ P1 was performed on a supercoiled plasmid template (using transcription buffer containing $30 \mathrm{mM}$ $\mathrm{NaCl}$; see Materials and Methods) in the presence of different concentrations of purified hexa-histidine-tagged DksA (Paul et al. 2004a), added at the indicated concentrations. Transcription by RNAP holoenzyme made from overproduction of $\alpha_{2} \beta \beta^{\prime}$ (black bars) or $\alpha_{2} \beta \beta^{\prime}$ and $\omega$ (gray bars) at each DksA concentration is expressed relative to transcription without DksA. Pairwise comparisons of transcription in the presence of different concentrations of DksA yielded a ratio of $1.08 \pm 0.19$ for RNAP $\pm \omega$ (nine independent experiments). (B) Half-lives of rrnB P1 promoter-RNAP complexes \pm DksA were determined by a transcription-based assay (see Materials and Methods and legend for Fig. 1). (Open symbols) 0.5 mM DksA; (filled symbols) no DksA; (triangles) RNAP made from overproduction of $\alpha_{2} \beta \beta^{\prime}$; (circles) RNAP made from overproduction of $\alpha_{2} \beta \beta^{\prime}$ and $\omega$. Two experiments were performed, and a representative one is shown. DksA decreased the lifetime of the complex an average of $3.0 \pm 0.02$-fold in the absence of $\omega$ and $4.7 \pm 1.0$-fold in the presence of $\omega$. (C) Half-lives of lacUV5 promoter-RNAP complexes \pm DksA were determined by a filter-binding assay (see Materials and Methods and legend for Fig. 3). (Open symbols) 0.5 mM DksA; (filled symbols) no DksA; (triangles) RNAP made from overproduction of $\alpha_{2} \beta \beta^{\prime}$; (circles) RNAP made from overproduction of $\alpha_{2} \beta \beta^{\prime}$ and $\omega$. Two experiments were performed, and a representative one is shown. DksA decreased the lifetime of the complex $8.5 \pm 1.4$-fold in the absence of $\omega$ and $6.1 \pm 0.6$-fold in the presence of $\omega$.
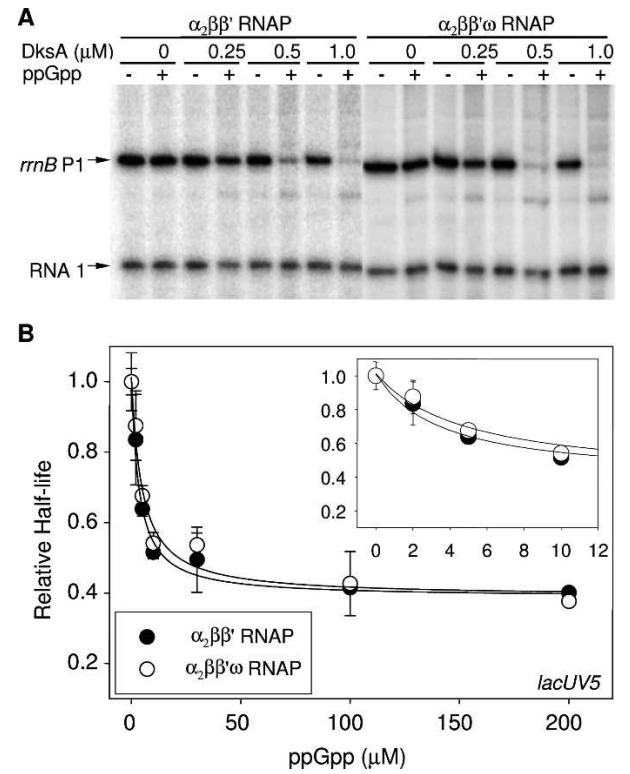

Figure 5. DksA rescues the ppGpp-sensitivity of RNAP lacking $\omega$. (A) Single-round transcription was performed on a supercoiled plasmid template containing the $\operatorname{rrnB} \mathrm{P} 1$ promoter. The reactions contained transcription buffer containing $30 \mathrm{mM} \mathrm{KCl}$; $400 \mu \mathrm{M}$ ppGpp and/or different concentrations of DksA were included as indicated. (Left panel) RNAP made by overproduction of $\alpha_{2} \beta \beta^{\prime}$ but not $\omega$. (Right panel) RNAP made by overproduction of $\alpha_{2} \beta \beta^{\prime}$ and $\omega$. (B) Lifetimes of RNAP-lacUV5 promoter complexes were determined in transcription buffer containing $100 \mathrm{mM} \mathrm{KCl}$ at different ppGpp concentrations in the presence of $0.5 \mu \mathrm{M}$ DksA using the filter-binding assay. (Filled circles) RNAP made by overproduction of $\alpha_{2} \beta \beta^{\prime}$ but not $\omega$; (open circles) RNAP made by co-overproduction of $\alpha_{2} \beta \beta^{\prime}$ and $\omega$. Halflives at each ppGpp concentration are plotted relative to the half-lives in the absence of ppGpp. Each fraction was generated by averaging the half-lives from several independent experiments. Error bars represent standard deviations from the mean.

function in vitro versus in vivo. We emphasize that although DksA can bypass the requirement for $\omega$ with respect to ppGpp function, the two proteins are not redundant: $\omega$ cannot substitute for DksA function in regulation of transcription initiation, and there is no evidence that DksA can substitute for $\omega$ function in RNAP assembly.

\section{Mechanism by which $\omega$ affects the response of RNAP to $p p G p p$}

The recent cocrystal of the T. thermophilus RNAP complex containing ppGpp defines a ppGpp-binding site adjacent to, but not overlapping, the active center (Artsimovitch et al. 2004). Assuming this is the functionally relevant binding site for ppGpp, the structure clearly indicates that $\omega$ and ppGpp are much too far apart to interact directly (Fig. 6A). Thus, the observed effect of $\omega$ on ppGpp function demonstrates that the proposed ppGppbinding region is affected by parts of the enzyme located relatively distant from the ppGpp-binding site. 

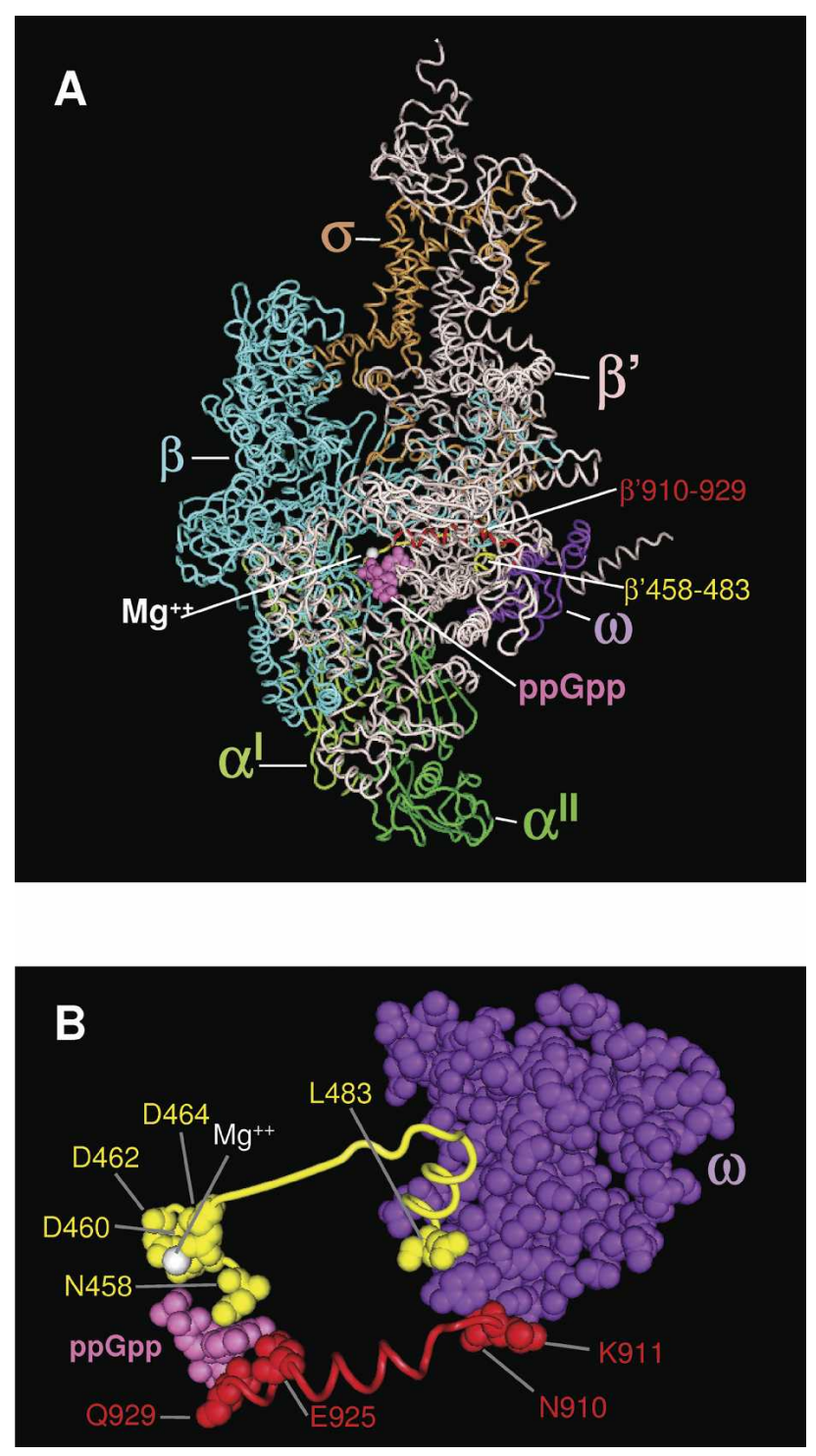

Figure 6. (A) Relative positions of $\omega$ and ppGpp in T. thermophilus RNAP holoenzyme. The model of the T. thermophilus ppGpp-RNAP structure, viewed from the entrance to the secondary channel, was constructed with PyMol (DeLano Scientific) from PDB coordinates 1 SMY (Artsimovitch et al. 2004). ppGpp is in magenta. Subunits are colored as follows: $\omega$, purple; $\alpha^{\mathrm{I}}$, light green; $\alpha^{\mathrm{II}}$, dark green; $\beta$, cyan; $\beta^{\prime}$, light pink; $\sigma$, orange. The catalytic $\mathrm{Mg}^{2+}$ is shown as a white ball. Two segments of $\beta^{\prime}$ connecting $\omega$ to the ppGpp-binding region (described in the text and enlarged below) are in red and yellow. (B) Potential communication between $\omega$ and the ppGpp-binding region. Only ppGpp (magenta), $\omega$ (purple), and two segments of $\beta^{\prime}$ connecting them (red and yellow) are shown. The complex has been rotated $90^{\circ}$ around the $X$-axis relative to the view in $A$. The same colors are used as in $A$. ppGpp, $\omega$, and $\beta^{\prime}$ residues of potential interest are in spacefill. $\beta^{\prime}$ residues (E. coli numbering) are indicated and were identified by alignment with T. thermophilus $\beta^{\prime}$. The corresponding $T$. thermophilus $\beta^{\prime}$ residues (in parentheses) are N458 (N737), D460 (D739), D462 (D741), D464 (D743), L483 (Q762), N910 (S1216), K911 (I1217), E925 (S1231), and Q929 (Q1235).
Effects of $\omega$ on ppGpp function could therefore involve (1) an allosteric effect of $\omega$ on ppGpp binding or (2) an allosteric effect of $\omega$ on ppGpp action. We note that there are two segments in $\beta^{\prime}$ (Fig. 6B) that directly connect $\omega$ with the ppGpp-binding site (see also Minakhin et al. 2001). Specifically, conserved region 1 of $\omega$ interacts with $\beta^{\prime}$ residues N910-K911 (amino acid numbering refers to E. coli RNAP). These residues are connected through a single $\alpha$-helix (red in Fig. 6A,B) to $\beta^{\prime}$ residues E925 and Q929, which, in turn, likely interact with ppGpp (Artsimovitch et al. 2004). $\omega$ also connects with the ppGpp-binding region through $\beta^{\prime}$ N458-L483 (yellow in Fig. 6A,B), in which L483 directly interacts with $\omega$; N458 interacts with the guanine base of ppGpp (Artsimovitch et al. 2004); D460, D462, and D464 coordinate a catalytic $\mathrm{Mg}^{2+}$; and D460 also helps coordinate a ppGppassociated $\mathrm{Mg}^{2+}$ (Artsimovitch et al. 2004).

We suggest that $\omega$ and the ppGpp-binding region might communicate through these segments in $\beta^{\prime}$, providing a possible structural explanation for the effect of $\omega$ on ppGpp function. For example, interactions of $\omega$ with $\beta^{\prime}$ might shift the orientation of the $\beta^{\prime}$ N910-Q929 $\alpha$-helix, facilitating ppGpp binding by altering the position of Q929 relative to ppGpp (model 1). Alternatively, $\omega$-dependent rearrangements in either (or both) of the segments of $\beta^{\prime}$ connecting $\omega$ and the ppGpp-binding region might alter the stabilities of kinetic intermediates on the pathway to open complex formation/dissociation, amplifying effects of ppGpp (model 2). In this context, we note that bacteriophage N4 single-stranded binding protein (N4SSB), an activator of N4 late gene transcription that facilitates an isomerization step on the pathway to open complex formation (Miller et al. 1997), functions by interacting with a segment of $\beta^{\prime}$ in contact with $\omega$, possibly through a similar allosteric communication mechanism.

\section{Mechanism by which DksA affects the response of RNAP to ppGpp}

The overall structure of DksA resembles that of the bacterial transcription elongation factors, GreA and GreB (Perederina et al. 2004). Like the Gre factors, DksA binds in the secondary channel of RNAP (Perederina et al. 2004; I. Toulokhonov, J. Mukhopadhyay, R.H. Ebright, and R.L. Gourse, unpubl.). There is currently no structure-based model that fully explains the effects of DksA on transcription initiation and its synergy with ppGpp. However, DksA rescues the effect of ppGpp on RNAP lacking $\omega$ and thus must facilitate binding or function of ppGpp, either directly or allosterically. For example, DksA could potentially facilitate binding or function of ppGpp by interacting with ppGpp directly in the RNAP secondary channel, as proposed previously (Perederina et al. 2004). Alternatively, DksA could facilitate ppGpp binding or function allosterically by working through the pathway of structural communication between $\omega$ and the ppGpp-binding site proposed above, potentially enhancing ppGpp binding or function by the same mechanism as $\omega$. Protein-protein footprinting experiments (I. Toulokhonov and R.L. Gourse, unpubl.) indi- 
cate that DksA, positioned in the secondary channel, is in close proximity to residues in the segments of $\beta^{\prime}$ linking $\omega$ and ppGpp.

We also note that higher concentrations of DksA on its own (i.e., in the absence of ppGpp and $\omega$ ) can directly inhibit or stimulate transcription initiation, depending on the kinetic characteristics of the promoter (Fig. 4; Paul et al. 2004a, 2005). In this case, DksA bypasses requirements for both ppGpp and $\omega$. One simple model is that DksA affects RNAP-promoter lifetime and transcription initiation through interactions with one or both of the segments of $\beta^{\prime}$ connecting $\omega$ and the ppGppbinding region.

\section{Additional roles for $\omega$ in vivo}

As indicated above, a strain lacking both $r p o Z$ and $d k s A$ is viable, but its growth is impaired, and it has nutritional requirements in addition to those resulting from mutations inactivating either $d k s A$ or $r p o Z$ alone (data not shown). It is possible that the additional phenotypes of the double mutant reflect some further impairment in the response of RNAP to ppGpp (by virtue of the loss of both DksA and $\omega)$. Alternatively, additional effects of the double mutant may be unrelated to ppGpp function. As noted above, $\omega$ has effects on transcription in the absence of ppGpp (R. Saecker and M.T. Record Jr., pers. comm.).

Finally, antibiotic production and morphogenesis in Streptomycetes sp. are positively controlled by ppGpp (Bibb 2005). Disruption of rpoZ drastically disrupts these processes in Streptomyces kasugaensis (Kojima et al. 2002), suggesting that the effects of ppGpp on RNAP in this organism are dependent on $\omega$ in vivo, perhaps by the same mechanism described here for E. coli.

\section{Materials and methods}

\section{Purification of overproduced $\alpha_{2} \beta \beta^{\prime}$ RNAP}

Holoenzyme prepared by purification of core RNAP after overproduction of $\alpha, \beta$, and $\beta^{\prime}$ in vivo, followed by reconstitution with purified $\sigma^{70}$, is referred to as "overproduced $\alpha_{2} \beta \beta^{\prime}$ RNAP"; this RNAP contains only trace amounts of $\omega$ (derived from the chromosomal rpoZ gene). Overproduced $\alpha_{2} \beta \beta^{\prime}$ RNAP was made using plasmid pIA299 (Artsimovitch et al. 2003), which carries E. coli rpo $A, r p o B$, and $r p o C$ fused to a $\mathrm{T} 7$ promoter. $r p o C$ in this vector codes for a $\beta^{\prime}$ subunit with a C-terminal hexa-histidine tag to facilitate purification. RLG7650 (BL21入DE3 transformed with pIA299) was grown at $30^{\circ} \mathrm{C}$ in $2 \mathrm{~L}$ of LB medium with 100 $\mu \mathrm{g} / \mathrm{mL}$ ampicillin to an $\mathrm{OD}_{600}$ of 0.35 and induced for $3 \mathrm{~h}$ by addition of $1 \mathrm{mM}$ IPTG. Cells were pelleted and lysed in grinding buffer (50 mM Tris at $\mathrm{pH}$ 8.0, 5\% glycerol, 2 mM EDTA, 233 $\mathrm{mM} \mathrm{NaCl}, 1 \mathrm{mM} \beta$-mercaptoethanol, $0.1 \mathrm{mM}$ DTT, $0.26 \mathrm{mM}$ PMSF) by sonication. RNAP was purified by Polymin P precipitation followed by $\mathrm{NH}_{4} \mathrm{SO}_{4}$ precipitation (Burgess and Jendrisak 1975), and the RNAP-containing pellet was resuspended in 10 $\mathrm{mL}$ of binding buffer $(40 \mathrm{mM}$ Tris- $\mathrm{HCl}$ at $\mathrm{pH} 8.0,500 \mathrm{mM} \mathrm{KCl}$, $5 \%$ glycerol, $0.1 \%$ Tween 20 ), loaded onto a $1-\mathrm{mL}$ column of Ni-NTA affinity resin (QIAGEN), washed with 4 column volumes of binding buffer, and eluted with binding buffer containing $300 \mathrm{mM}$ imidazole. Fractions containing RNAP were pooled and diluted to a final concentration of $200 \mathrm{mM} \mathrm{KCl}$, and DTT was added to $2 \mathrm{mM}$. Protein was loaded onto a 1-mL Heparin Hi-Trap column (Amersham Biosciences) equilibrated with TGE (10 mM Tris- $\mathrm{HCl}$ at $\mathrm{pH}$ 8.0, $0.1 \mathrm{mM}$ EDTA, 5\% glycerol) containing $200 \mathrm{mM} \mathrm{NaCl}$; the column was washed with 10 column volumes of TGE containing $200 \mathrm{mM} \mathrm{NaCl}$; and RNAP was eluted with TGE containing $600 \mathrm{mM} \mathrm{NaCl}$. The purity of the RNAP was estimated at $>99 \%$ by SDS-PAGE. Only a very faint band corresponding to the position of $\omega$ was detected in the overproduced $\alpha_{2} \beta \beta^{\prime}$ RNAP, presumably deriving from trace amounts of host-encoded $\omega$ that assembled with the overproduced core subunits. Purified $\sigma^{70}$ was added to overproduced core enzyme in two- to sixfold molar excess by incubation at $30^{\circ} \mathrm{C}-37^{\circ} \mathrm{C}$ for $60 \mathrm{~min}$.

\section{Purification of overproduced $\alpha \beta \beta^{\prime} \omega$ RNAP}

Holoenzyme purified after overproduction of not only $\alpha, \beta$, and $\beta^{\prime}$ but also $\omega$ in vivo, followed by reconstitution with purified $\sigma^{70}$, is referred to as "overproduced $\alpha_{2} \beta \beta^{\prime} \omega$ RNAP." Overpro-

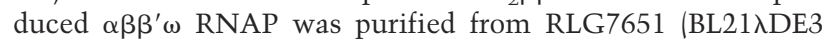
containing both pIA299, which overproduces $\alpha_{2} \beta \beta^{\prime}$, and pCDF $\omega$, which overproduces $\omega)$. pCDF $\omega$ was constructed by insertion of the E. coli rpoZ gene between the NdeI and XhoI sites of pCDF-1 (Novagen) (Y. Kim and R.H. Ebright, unpubl.). RLG7651 was grown in $2 \mathrm{~L}$ of LB with $100 \mu \mathrm{g} / \mathrm{mL}$ ampicillin and $50 \mu \mathrm{g} / \mathrm{mL}$ spectinomycin at $30^{\circ} \mathrm{C}$ to an $\mathrm{OD}_{600}$ of 0.35 and induced for $3 \mathrm{~h}$ with $1 \mathrm{mM}$ IPTG. Overproduced $\alpha_{2} \beta \beta^{\prime} \omega$ RNAP was purified exactly as described above for overproduced $\alpha_{2} \beta \beta^{\prime}$ RNAP; the two preparations were purified in parallel and were of similar purity as assessed by SDS-PAGE stained with Coomassie blue or silver. $\omega$ was a prominent band in SDS-PAGE of the overproduced $\alpha_{2} \beta \beta^{\prime} \omega$ RNAP.

\section{Purification of $\omega$ and reconstitution with core RNAP in vitro}

Holoenzyme prepared by purification of core RNAP after overproduction of $\alpha, \beta$, and $\beta^{\prime}$ in vivo, followed by reconstitution with purified $\omega$ and $\sigma^{70}$ in vitro, is referred to as "overproduced $\alpha_{2} \beta \beta^{\prime}+\omega$ RNAP." Native (untagged) $\omega$ was purified from $2 \mathrm{~L}$ of BL2 $1 \lambda$ DE3 carrying pCDF $\omega$ grown at $30^{\circ} \mathrm{C}$ in LB with $50 \mu \mathrm{g} / \mathrm{mL}$ spectinomycin to an $\mathrm{OD}_{600}$ of 0.4-0.6 and induced for $2 \mathrm{~h}$ with 1 mM IPTG. $\omega$ was purified as described (Gentry and Burgess 1990). Since the majority of $\omega$ remained in the soluble fraction, only the protocol relevant to the soluble fraction was used. Furthermore, since $\omega$ was $>99 \%$ pure after the Q-Sepharose Fast Flow and Red-Sepharose column steps, the final Mono-Q chromatographic step was omitted. Overproduced $\alpha_{2} \beta \beta^{\prime}$ RNAP core was incubated at $37^{\circ} \mathrm{C}$ with fivefold to 10 -fold molar excess of $\omega$ in $1.5 \times$ transcription buffer and either 45 or $250 \mathrm{mM} \mathrm{NaCl}$ for 20 min as described (Gentry and Burgess 1990) before addition of two- to fivefold molar excess of $\sigma$ for $40 \mathrm{~min}$. Although we did not determine the stoichiometry of $\omega$ :RNAP, the response to ppGpp of RNAP reconstituted with $\omega$ in vitro was within error of that of overexpressed RNAP with overproduced $\omega$ (Table 1), suggesting that these preparations were saturated with $\omega$ to the same extent. We note that incubation of $\omega$ with overproduced $\alpha_{2} \beta \beta^{\prime}$ RNAP in a buffer containing $50 \%$ glycerol resulted in only partial restoration of ppGpp sensitivity to RNAP, suggesting that glycerol might inhibit the rate of association of $\omega$ with core.

\section{Purification of native RNAP}

Holoenzyme purified without overproduction from cells wildtype for rpoZ is referred to as "native," and holoenzyme purified 
from $r p o Z::$ kan cells without overproduction is referred to as "native (rpoZ::kan)." Native holoenzymes were purified by standard methods (Burgess and Jendrisak 1975) or by immunoaffinity chromatography using the polyol-responsive antibody NT73 (Neoclone) as described (Thompson et al. 1992). The purities of the resulting holoenzymes were confirmed by SDSPAGE. No $\omega$ protein was detected in the preparation made from the rpoZ mutant strain.

\section{Transcription assays}

Multiple-round assays were carried out with 10-30 nM RNAP in transcription buffer $(40 \mathrm{mM}$ Tris- $\mathrm{HCl}$ at $\mathrm{pH} 7.9,10 \mathrm{mM}$ $\mathrm{MgCl}_{2}, 1 \mathrm{mM} \mathrm{DTT}, 0.1 \mathrm{mg} / \mathrm{mL}$ BSA, and $\mathrm{KCl}$ or $\mathrm{NaCl}$ at the concentrations indicated in the figure legends) at $30^{\circ} \mathrm{C}$ (Ross et al. 1990). Reactions containing transcription buffer, NTPs (200 $\mu \mathrm{M}$ ATP, GTP, and CTP; $10 \mu \mathrm{M}$ UTP; and $1 \mu \mathrm{Ci}$ of $\left.\left[\alpha-{ }^{32} \mathrm{UTP}\right]\right)$, and $r r n B$ P1 template (with $400 \mu \mathrm{M}$ ppGpp or $0.5 \mu \mathrm{M}$ DksA or both as indicated) were initiated by addition of RNAP and stopped after $10 \mathrm{~min}$. For single-round reactions, the template was incubated with RNAP for $10 \mathrm{~min}, 10 \mu \mathrm{g} / \mathrm{mL}$ heparin was added, and after $10 \mathrm{sec}$ transcription was initiated by addition of NTPs. In addition, 0-400 $\mu \mathrm{M}$ ppGpp and 0.2-1.0 $\mu \mathrm{M}$ DksA were included when indicated. Reactions were terminated by addition of an equal volume of urea stop buffer (Ross et al. 1990) after $10 \mathrm{~min}$, followed by analysis by gel electrophoresis and phosphorimaging.

\section{Dissociation kinetics}

Half-lives of open complexes were determined from semilog plots of fraction remaining versus time. Dissociation rate constants, $\mathrm{k}_{\mathrm{d}}$, were determined from the first-order decay equation, $\mathrm{cpm}_{\text {retained }}=\left(\mathrm{cpm}_{\max }\right) \mathrm{e}^{-\mathrm{k}} \mathrm{d}^{\mathrm{t}}$. Half-lives of RNAP on $\operatorname{rrnB}$ P1 were measured by a transcription-based assay (Barker et al. 2001b). Supercoiled plasmid pRLG6798 (0.5 nM), containing the $\operatorname{rrn} B$ P1 promoter ( -66 to +50 end points), was incubated with 10-30 nM RNAP in transcription buffer (see above; $30 \mathrm{mM} \mathrm{KCl)} \mathrm{for} 10$ min at $30^{\circ} \mathrm{C}$ to form complexes, and $400 \mu \mathrm{M}$ ppGpp (Tri-link, Inc.) and 0.2-1.0 $\mu \mathrm{M}$ DksA were included when indicated. After addition of $10 \mu \mathrm{g} / \mathrm{mL}$ heparin, samples were removed at intervals, and transcription was initiated by addition of NTPs and allowed to proceed for $10 \mathrm{~min}$. Half-lives of RNAP on the lacUV5 promoter were measured by filter-binding (Ross and Gourse 2005). lacUV5 promoter fragments (end points -140 to +88 ) from pRLG4264 (Ross and Gourse 2005) were incubated for $10 \mathrm{~min}$ at $30^{\circ} \mathrm{C}$ with $5-10 \mathrm{nM}$ RNAP in transcription buffer containing $100 \mathrm{mM} \mathrm{KCl}$, and $0.5 \mu \mathrm{M}$ DksA and/or 2-400 $\mu \mathrm{M}$ ppGpp were added as indicated before RNAP addition. After addition of $10 \mu \mathrm{g} / \mathrm{mL}$ heparin, samples were removed at intervals and filtered through nitrocellulose disks. The filter-bound complexes were washed with $100 \mathrm{mM} \mathrm{NaCl}, 10 \mathrm{mM}$ Tris- $\mathrm{HCl}$ (pH 8.0), and $1 \mathrm{mM}$ EDTA, dried, and counted with a Packard scintillation counter.

We emphasize that intrinsic lifetimes of promoter-RNAP complexes are very sensitive to slight variations in solution conditions, topology of DNA, or heparin concentrations. Therefore, RNAP preparations under comparison were always tested with the same solutions in the same experiment.

\section{DksA preparations}

$\mathrm{N}$-Terminal hexa-histidine-tagged DksA was prepared as described previously (Paul et al. 2004a). No band migrating at the position of $\omega$ was detected under conditions where $0.1 \%$ potential contamination with $\omega$ would have been detectable. Since
$500 \mathrm{nM}$ DksA was used in transcription reactions, $\omega$ could therefore have been present at no more than $0.5 \mathrm{nM}$, which would have been greatly substoichiometric with the 5-30 nM RNAP used.

\section{Acknowledgments}

We thank I. Artsimovitch, R. Burgess, Y. Kim, R. Landick, and R. Saecker for materials and/or discussion. This work was supported by research grants to R.L.G. (GM37048) and R.H.E. (GM41376) from the National Institutes of Health and by a predoctoral fellowship (to C.E.V.) and an investigatorship (to R.H.E.) from the Howard Hughes Medical Institute.

\section{References}

Artsimovitch, I., Svetlov, V., Murakami, K.S., and Landick, R. 2003. Co-overexpression of Escherichia coli RNA polymerase subunits allows isolation and analysis of mutant enzymes lacking lineage-specific sequence insertions. J. Biol. Chem. 278: 12344-12355.

Artsimovitch, I., Patlan, V., Sekine, S., Vassylyeva, M.N., Hosaka, T., Ochi, K., Yokoyama, S., and Vassylyev, D.G. 2004. Structural basis for transcription regulation by alarmone ppGpp. Cell 117: 299-310.

Barker, M.M., Gaal, T., and Gourse, R.L. 2001a. Mechanism of regulation of transcription initiation by ppGpp. II. Models for positive control based on properties of RNAP mutants and competition for RNAP. J. Mol. Biol. 305: 689-702.

Barker, M.M., Gaal, T., Josaitis, C.A., and Gourse, R.L. $2001 \mathrm{~b}$. Mechanism of regulation of transcription initiation by ppGpp. I. Effects of ppGpp on transcription initiation in vivo and in vitro. J. Mol. Biol. 305: 673-688.

Berghofer-Hochheimer, Y., Lu, C.Z., and Gross, C.A. 2005. Altering the interaction between $\sigma^{70}$ and RNA polymerase generates complexes with distinct transcription-elongation properties. Proc. Natl. Acad. Sci. 102: 1157-1162.

Bibb, M.J. 2005. Regulation of secondary metabolism in streptomycetes. Curr. Opin. Microbiol. 8: 208-215.

Burgess, R.R. and Jendrisak, J.J. 1975. A procedure for the rapid, large-scale purification of Escherichia coli DNA-dependent RNA polymerase involving Polymin $\mathrm{P}$ precipitation and DNA-cellulose chromatography. Biochemistry 14:46344638.

Cashel, M., Gentry, D.R., Hernandez, V.H., and Vinella, D. 1996. The stringent response. In Escherichia coli and Salmonella typhimurium: Cellular and molecular biology (ed. F.C. Neidhardt), pp. 1458-1496. ASM Press, Washington, DC.

Gentry, D.R. and Burgess, R.R. 1989. rpoZ, encoding the $\omega$ subunit of Escherichia coli RNA polymerase, is in the same operon as spoT. J. Bacteriol. 171: 1271-1277.

1990. Overproduction and purification of the $\omega$ subunit of Escherichia coli RNA polymerase. Protein Expr. Purif. 1: 81-86.

-1993. Cross-linking of Escherichia coli RNA polymerase subunits: Identification of $\beta^{\prime}$ as the binding site of $\omega$. Biochemistry 32: 11224-11227.

Gentry, D., Xiao, H., Burgess, R., and Cashel, M. 1991. The $\omega$ subunit of Escherichia coli K-12 RNA polymerase is not required for stringent RNA control in vivo. J. Bacteriol. 173: 3901-3903.

Geszvain, K.M. and Landick, R. 2004. The structure of bacterial RNA polymerase. In The bacterial chromosome (ed. N.P. Higgins), pp. 283-296. American Society for Microbiology, Washington, DC. 
Ghosh, P., Ishihama, A., and Chatterji, D. 2001. Escherichia coli RNA polymerase subunit $\omega$ and its $\mathrm{N}$-terminal domain bind full-length $\beta^{\prime}$ to facilitate incorporation into the $\alpha_{2} \beta$ subassembly. Eur. J. Biochem. 268: 4621-4627.

Ghosh, P., Ramakrishnan, C., and Chatterji, D. 2003. Inter-subunit recognition and manifestation of segmental mobility in Escherichia coli RNA polymerase: A case study with $\omega-\beta^{\prime}$ interaction. Biophys. Chem. 103: 223-237.

Gralla, J.D. 2005. Escherichia coli ribosomal RNA transcription: Regulatory roles for ppGpp, NTPs, architectural proteins and a polymerase-binding protein. Mol. Microbiol. 55: $973-977$.

Igarashi, K., Fujita, N., and Ishihama, A. 1989. Promoter selectivity of Escherichia coli RNA polymerase: $\omega$ factor is responsible for the ppGpp sensitivity. Nucleic Acids Res. 17: 8755-8765.

Kojima, I., Kasuga, K., Kobayashi, M., Fukasawa, A., Mizuno, S., Arisawa, A., and Akagawa, H. 2002. The rpoZ gene, encoding the RNA polymerase $\omega$ subunit, is required for antibiotic production and morphological differentiation in Streptomyces kasugaensis. J. Bacteriol. 184: 6417-6423.

Miller, A., Wood, D., Ebright, R.H., and Rothman-Denes, L.B. 1997. RNA polymerase $\beta^{\prime}$ subunit: A target of DNA bindingindependent activation [see comments]. Science 275: 16551657.

Minakhin, L., Bhagat, S., Brunning, A., Campbell, E.A., Darst, S.A., Ebright, R.H., and Severinov, K. 2001. Bacterial RNA polymerase subunit $\omega$ and eukaryotic RNA polymerase subunit RPB6 are sequence, structural, and functional homologs and promote RNA polymerase assembly. Proc. Natl. Acad. Sci. 98: 892-897.

Mukherjee, K. and Chatterji, D. 1997. Studies on the $\omega$ subunit of Escherichia coli RNA polymerase-Its role in the recovery of denatured enzyme activity. Eur. J. Biochem. 247: 884-889.

Mukherjee, K., Nagai, H., Shimamoto, N., and Chatterji, D. 1999. GroEL is involved in activation of Escherichia coli RNA polymerase devoid of the $\omega$ subunit in vivo. Eur. $J$. Biochem. 266: 228-235.

Murakami, K.S., Masuda, S., Campbell, E.A., Muzzin, O., and Darst, S.A. 2002a. Structural basis of transcription initiation: An RNA polymerase holoenzyme-DNA complex. Science 296: 1285-1290.

Murakami, K.S., Masuda, S., and Darst, S.A. 2002b. Structural basis of transcription initiation: RNA polymerase holoenzyme at $4 \AA$ resolution. Science 296: 1280-1284.

Paul, B.J., Barker, M.M., Ross, W., Schneider, D.A., Webb, C., Foster, J.W., and Gourse, R.L. 2004a. DksA: A critical component of the transcription initiation machinery that potentiates the regulation of rRNA promoters by ppGpp and the initiating NTP. Cell 118: 311-322.

Paul, B.J., Ross, W., Gaal, T., and Gourse, R.L. 2004b. rRNA transcription in Escherichia coli. Annu. Rev. Genet. 38: 749-770.

Paul, B.J., Berkmen, M.B., and Gourse, R.L. 2005. DksA potentiates direct activation of amino acid promoters by ppGpp. Proc. Nat1. Acad. Sci. 102: 7823-7828.

Perederina, A., Svetlov, V., Vassylyeva, M.N., Tahirov, T.H., Yokoyama, S., Artsimovitch, I., and Vassylyev, D.G. 2004. Regulation through the secondary channel-Structural framework for ppGpp-DksA synergism during transcription. Cell 118: 297-309.

Ross, W. and Gourse, R.L. 2005. Sequence-independent upstream DNA- $\alpha$ CTD interactions strongly stimulate Escherichia coli RNA polymerase-lacUV5 promoter association. Proc. Nat1. Acad. Sci. 102: 291-296.
Ross, W., Thompson, J.F., Newlands, J.T., and Gourse, R.L. 1990. E. coli Fis protein activates ribosomal RNA transcription in vitro and in vivo. EMBO J. 9: 3733-3742.

Tang, H., Severinov, K., Goldfarb, A., and Ebright, R.H. 1995. Rapid RNA polymerase genetics: One-day, no-column preparation of reconstituted recombinant Escherichia coli RNA polymerase. Proc. Nat1. Acad. Sci. 92: 4902-4906.

Thompson, N.E., Hager, D.A., and Burgess, R.R. 1992. Isolation and characterization of a polyol-responsive monoclonal antibody useful for gentle purification of Escherichia coli RNA polymerase. Biochemistry 31: 7003-7008.

Vassylyev, D.G., Sekine, S., Laptenko, O., Lee, J., Vassylyeva, M.N., Borukhov, S., and Yokoyama, S. 2002. Crystal structure of a bacterial RNA polymerase holoenzyme at $2.6 \AA$ resolution. Nature 417: 712-719.

Xiao, H., Kalman, M., Ikehara, K., Zemel, S., Glaser, G., and Cashel, M. 1991. Residual guanosine 3',5'-bispyrophosphate synthetic activity of relA null mutants can be eliminated by spoT null mutations. J. Biol. Chem. 266: 5980-5990. 


\section{Erratum}

Genes \& Development 19: 2378-2387 (2005)

Response of RNA polymerase to ppGpp: requirement for the $\omega$ subunit and relief of this requirement by DksA Catherine E. Vrentas, Tamas Gaal, Wilma Ross, Richard H. Ebright, and Richard L. Gourse

In the print version of the above-mentioned paper, the DOI number was incorrect. The correct DOI line for this paper should read:

Article and publication are at http://www.genesdev.org/cgi/doi/10.1101/gad.1340305. 


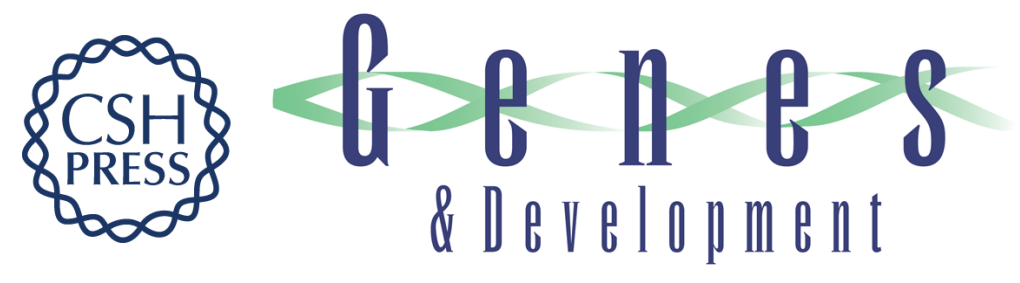

\section{Response of RNA polymerase to ppGpp: requirement for the $\omega$ subunit and relief of this requirement by DksA}

Catherine E. Vrentas, Tamas Gaal, Wilma Ross, et al.

Genes Dev. 2005, 19:

Access the most recent version at doi:10.1101/gad.1340305

Related Content

References

\section{License}

Email Alerting Service
This article cites 32 articles, 13 of which can be accessed free at:

http://genesdev.cshlp.org/content/19/19/2378.full.html\#ref-list-1

Articles cited in:

http://genesdev.cshlp.org/content/19/19/2378.full.html\#related-urls

Errata for vol. 19, p. 2378

Genes Dev. November , 2005 19: 2644

Receive free email alerts when new articles cite this article - sign up in the box at the top right corner of the article or click here.

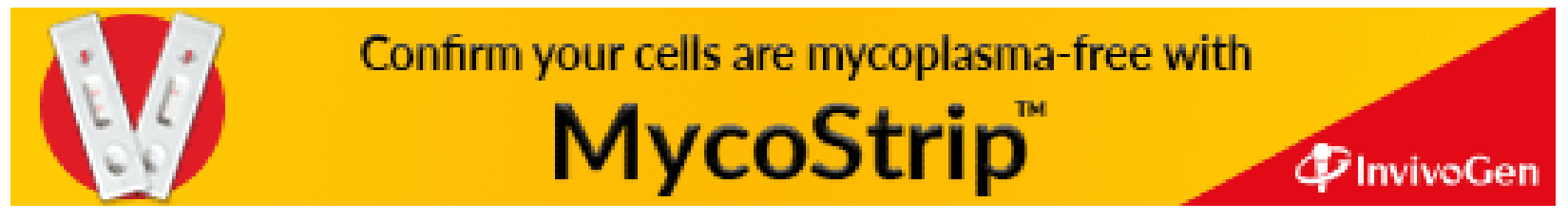

